\title{
Force and Trajectory Control of Industrial Robots in Stiff Contact
}

\author{
Friedrich Lange, Wieland Bertleff, and Michael Suppa
}

\begin{abstract}
Position-based force control is presented, incorporating compliance in the robot joints and possibly in a force- / torque-sensor and/or the environment. First, the total compliance is identified. Then, in the control phase, the desired pose of the tool center point is computed from the force control error. Thus standard position control may be applied. This leads to an inherently stable control scheme, even with a low sampling rate of the sensor interface and unknown environmental compliance. The method is designed for applications of industrial robots, e.g. assembly tasks. Parallel control considers the existence of a reference trajectory which allows feedforward in force controlled directions. The paper further examines couplings between forces and torques, which are important for partially constrained configurations. A possible impact force is considered when colliding with an unexpected object.
\end{abstract}

\section{INTRODUCTION}

Modern force control applications predominantly use impedance-based schemes [1] which result in a more intuitive trajectory of the robot end-effector [2], [3]. This is particularly desired for tasks in which a robot interferes with a human, e.g. when moving or teaching the robot by holding an end-effector fixed handle which is connected to the robot by a force- / torque-sensor. But there are also applications which require an explicit force control scheme since otherwise intolerable forces would occur [4], [5]. This is typically the case when the robot is in contact with a stiff environment, e.g. in assembly tasks.

Another approach to deal with stiff contacts is the use of mechanical compliances since this tolerates low bandwidth controllers or large communication times. Therefore compliant devices have been used in the first force control applications [6]. Still they are proposed for the assembly in moving belt production since conveyors may show a bucking that cannot be tracked with small error by a heavy robot [7]. Today, compliance is integrated within a force- / torque sensor, which besides the prevention of (limited) high bandwidth force errors allows the compensation of large scale but low bandwidth force errors. A typical compliant sensor is offered by SCHUNK ${ }^{\circledR}$ [8], [9]. In contrast to strain gauge sensing in usual stiff sensors [10], this sensor is realized by position sensitive devices (PSD) to measure the deflections of springs between the robot and the end-effector. Typical deflections of this sensor are $1 \mathrm{~mm}$ or $1 \mathrm{deg}$ at the robot flange. This, however, may result in unfavorable deflections or even oscillations when the end-effector is dynamically moved without contact. Therefore, advanced force control applications try to minimize additional compliance.

The authors are with the German Aerospace Center (DLR), Robotics and Mechatronics Center (RMC), D-82234 Wessling, Germany friedrich. langeddlr.de

This work is partially funded by KUKA Laboratories $\mathrm{GmbH}$
High performance force control schemes act directly on the motor torques of the robot axes [11]. Unfortunately, this is not supported by typical industrial robot controllers. Instead, a position interface is usually provided. This motivates force control as an outer loop to the inner position loop [12], [13]. Beyond that, force control can be understood as the control of displacements that are caused by the forces. With a compliant sensor this has been proposed by the authors and results in a fast reduction of force errors when approaching an unexpected object [14]. But the method can also be applied with a stiff sensor. In this case, in contrast to [14], other significant compliances in the robot and the environment have to be incorporated. This paper therefore presents a position-based approach for force- / torque control, which does not require a dominant compliance within the sensor.

It is not needed that the environmental characteristics are known. Disregarded environmental compliance may cause instability when using motor torques as controller output [3], [4], [15]. Instead, for position-based force control, additional compliance acts stabilizing on the system [16]. So the assumption of zero environmental compliance as in [14] is a sufficient supposition for stability.

In contrast to previous assumptions of the authors, the compliance of typical industrial robots, end-effectors, and environments is often large enough to downsize the aforementioned high bandwidth force errors. So force control can solve assembly problems with low tolerance, even without

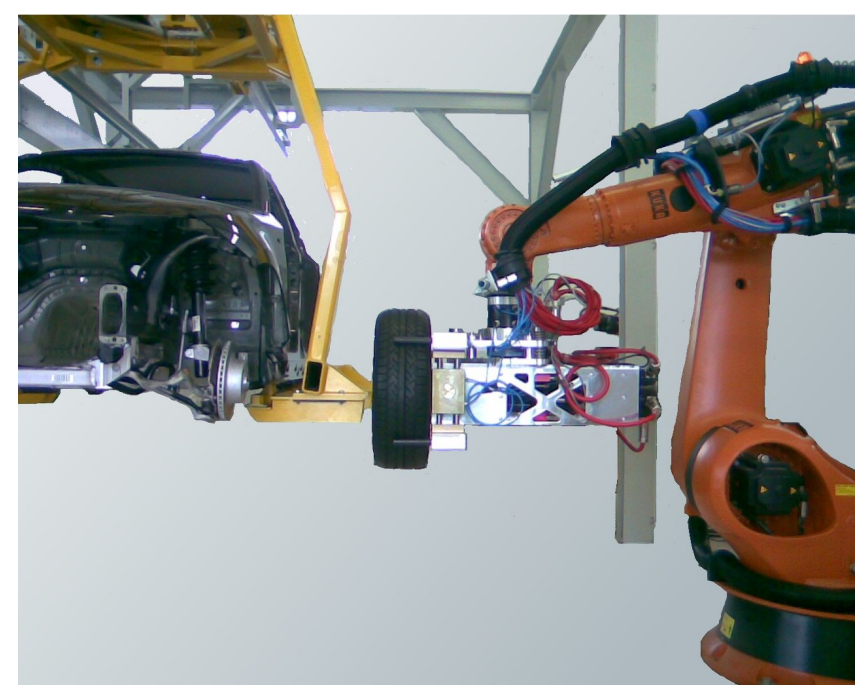

Fig. 1. Set-up at $i w b$ for mounting wheels to a moving car body using a compliantly suspended end-effector. 
additional compliance. This is the motivation for a review of the wheel assembly task (Fig. 1) which has been executed by the authors using a compliantly suspended end-effector [17]. Even more since this task has been shown without force control in [18] and [19]. There, the dominant compliance seems to be the power \& free conveyor, a rail in which the car body is suspended.

The paper is organized as follows: In section II all kinds of compliances are modeled. Then the force control scheme is summarized in section III, followed by some implementation details. Section IV discusses the approach of parallel control, an overlay of the tracking of a given trajectory and force controlled deviations. Section V explains the effect of couplings and of special contact formations. Finally, sections VI to VIII provide experimental results, the conclusion, and an outlook.

\section{Modeling}

For position-based force control, modeling means the formulation of a rule that computes a positional or orientational increment that results in the desired change of the force or the torque. Unless otherwise noted, in this section only the static relation is concerned, since velocities and accelerations are rather small in force control and therefore they can be disregarded usually.

\section{A. Modeling of the Robot}

In this section the robot is assumed to have stiff links but compliant axes (joints) $\mathbf{q}$ where the latter are compliant only in the axis direction. The kinematic transforms of the robot are given and compute the Cartesian pose $\mathbf{x}$ of the tool center point (tcp) with respect to the world system. The parameters are known. Inaccuracies in the latter assumptions have only marginal influence on the following equations.

External forces and torques $\mathbf{f}$ affect the joint torques $\tau$ by

$$
\tau=\mathbf{J}^{T} \mathbf{f},
$$

where $\mathbf{J}$ is the Jacobian of the robot. $\mathbf{f}$ is the vector of forces and torques at the tcp, expressed with respect to the world frame.

Assuming a (usually diagonal) joint compliance matrix $\mathbf{C}_{q}$, the joint displacements $\Delta \mathbf{q}$ that are caused by the forces and torques at the tcp are

$$
\Delta \mathbf{q}=\mathbf{C}_{q} \tau .
$$
is

Then the resulting Cartesian displacement $\Delta \mathbf{x}_{j}$ at the tcp

$$
\Delta \mathbf{x}_{j}=\mathbf{J} \boldsymbol{\Delta} \mathbf{q}
$$

which results in

$$
\begin{aligned}
\Delta \mathbf{x}_{j} & =\mathbf{J C}_{q} \mathbf{J}^{T} \mathbf{f} . \\
\mathbf{C}_{j} & =\mathbf{J C}_{q} \mathbf{J}^{T}
\end{aligned}
$$

represents that part of the total compliance at the tcp, that is due to the compliance in the robot joints.

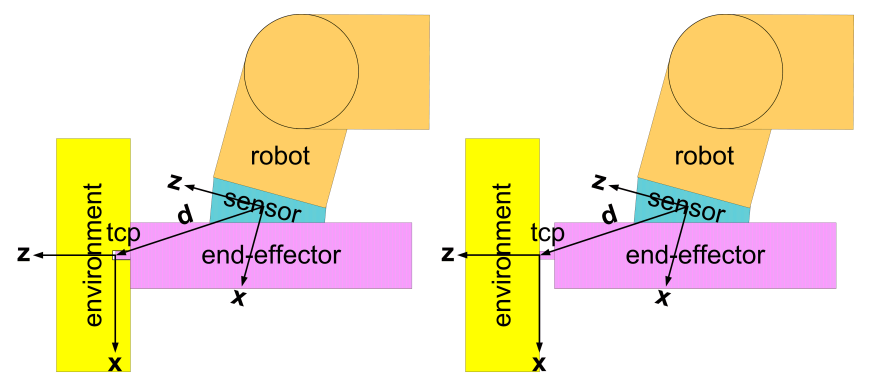

Fig. 2. Fully (left) and partially (right) constrained setup.

It should be noted, that this formulation neglects velocity or acceleration dependent terms, that might be significant since e.g. the inertial forces of the robot links can be higher than the contact forces. This is especially important in the transition from free to constrained motion, since in free motion there may be a significant speed. This will be further discussed in section III-C.

\section{B. Modeling of the Sensor}

The tcp is assumed to be located at a distance $\mathbf{d}$ from the sensor, where $\mathbf{d}$ is expressed in the sensor system, which, besides deflections, has the same orientation as the tcp system (Fig. 2). The sensor is modeled by a translational and a rotational spring with compliances ${ }_{s} \mathbf{C}_{p}$ and ${ }_{s} \mathbf{C}_{\varphi}$. Then the equations are set separately for the forces ${ }_{t} \mathbf{f}_{p}$ and the torques ${ }_{t} \mathbf{f}_{\varphi}$ and thus for the Cartesian positional and orientational increments $\boldsymbol{\Delta}_{t} \mathbf{x}_{p}$ and $\boldsymbol{\Delta}_{t} \mathbf{x}_{\varphi}$, all with respect to the tcp instead of the world frame.

According to the wrench equations, the forces and torques at the sensor, ${ }_{s} \mathbf{f}_{p}$ and ${ }_{s} \mathbf{f}_{\varphi}$, are

$$
\begin{aligned}
{ }_{s} \mathbf{f}_{p} & ={ }_{t} \mathbf{f}_{p} \\
{ }_{s} \mathbf{f}_{\varphi} & ={ }_{t} \mathbf{f}_{\varphi}+\mathbf{d} \times{ }_{t} \mathbf{f}_{p} .
\end{aligned}
$$

Then, assuming (usually diagonal) compliances in the sensor ${ }_{s} \mathbf{C}_{p},{ }_{s} \mathbf{C}_{\varphi}$, the displacements in the sensor are

$$
\begin{aligned}
\boldsymbol{\Delta}_{s} \mathbf{x}_{p} & ={ }_{s} \mathbf{C}_{p} \cdot{ }_{s} \mathbf{f}_{p} \\
\boldsymbol{\Delta}_{s} \mathbf{x}_{\varphi} & ={ }_{s} \mathbf{C}_{\varphi} \cdot{ }_{s} \mathbf{f}_{\varphi} .
\end{aligned}
$$

The pose increments at the tcp are then computed by the twist equations

$$
\begin{aligned}
\boldsymbol{\Delta}_{t} \mathbf{x}_{p} & =\boldsymbol{\Delta}_{s} \mathbf{x}_{p}-\mathbf{d} \times \boldsymbol{\Delta}_{s} \mathbf{x}_{\varphi} \\
\boldsymbol{\Delta}_{t} \mathbf{x}_{\varphi} & =\boldsymbol{\Delta}_{s} \mathbf{x}_{\varphi},
\end{aligned}
$$

or, more accurately, by a transform using the corresponding homogeneous matrices.

This can be merged to

$$
\boldsymbol{\Delta}_{t} \mathbf{x}_{s}={ }_{t} \mathbf{C}_{s} \cdot{ }_{t} \mathbf{f}
$$

with

$$
{ }_{t} \mathbf{C}_{s}=\left[\begin{array}{cc}
{ }_{s} \mathbf{C}_{p}-\tilde{\mathbf{d}}{ }_{s} \mathbf{C}_{\varphi} \tilde{\mathbf{d}} & -\tilde{\mathbf{d}}_{s} \mathbf{C}_{\varphi} \\
{ }_{s} \mathbf{C}_{\varphi} \tilde{\mathbf{d}} & { }_{s} \mathbf{C}_{\varphi}
\end{array}\right]
$$

where

$$
\tilde{\mathbf{d}}=\left[\begin{array}{ccc}
0 & -d_{z} & d_{y} \\
d_{z} & 0 & -d_{x} \\
-d_{y} & d_{x} & 0
\end{array}\right]
$$




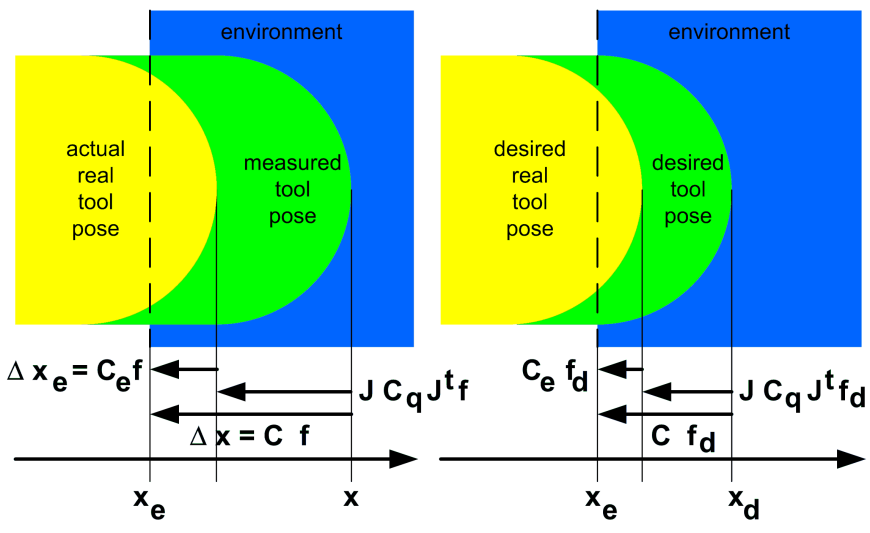

Fig. 3. Actual and desired forces and deflections with a round tool tip pressing against a static environment, assuming no sensor compliance.

With respect to the world frame, (12) can be expressed by

$$
\Delta \mathbf{x}_{s}=\mathbf{C}_{s} \cdot \mathbf{f}=\mathbf{R}^{\prime} \cdot{ }_{t} \mathbf{C}_{s} \cdot \mathbf{R}^{\prime T} \cdot \mathbf{f},
$$

where $\mathbf{R}^{\prime}$ is the $6 \times 6$ extended rotation matrix of the pose of the tcp with respect to the world frame.

$\mathbf{R}^{\prime}$ depends on the representation of the orientation within the pose vector. For a usual definition by Cardan angles it can be set to $\mathbf{R}^{\prime}=\operatorname{diag}(\mathbf{R}, \mathbf{R})$, as the fourth element of $\mathbf{x}$ represents the rotation around the $x$-axis etc.. $\mathbf{R}$ is the normal $3 \times 3$ rotation matrix. ${ }^{1}$

$\Delta \mathbf{x}_{s}$ in (15) represents the deflection at the tcp that is caused by a force at the tcp and compliance in the sensor.

These equations can also be used with a stiff sensor, then with the sensor compliance ${ }_{s} \mathbf{C}_{p},{ }_{s} \mathbf{C}_{\varphi}$ close or equal to zero.

Other concentrated sources of compliance can be treated similarly, e.g. a weak part of the end-effector.

\section{Modeling of the Environment}

A special case is compliance that is expressed with respect to the contact point $(\mathbf{d}=\mathbf{0})$, usually referred as environmental compliance $\mathbf{C}_{e}$ that needs no transformation.

$$
\Delta \mathbf{x}_{e}=\mathbf{C}_{e} \mathbf{f}=\left[\begin{array}{cc}
\mathbf{C}_{e p} & 0 \\
0 & \mathbf{C}_{e \varphi}
\end{array}\right]\left[\begin{array}{l}
\mathbf{f}_{p} \\
\mathbf{f}_{\varphi}
\end{array}\right]
$$

Here it is assumed, that the tcp and the contact point coincide. The contact pose $\mathbf{x}_{e}$ is defined by the real contact pose for the case with zero force. Otherwise the real contact pose is deflected by $-\Delta \mathbf{x}_{e}$ with respect to there (see Fig. 3).

A more refined modeling may be possible according to the characteristics of the environment, e.g. for the application of Fig. 1, where the car body is suspended in the power \& free conveyor. Then, first, the inertia of the car body can be considered, and, second, the steady state compliance can be derived from a rotational deviation at the suspension and the weight of the car acting at the center of gravity. This can be considered differently for the components in and normal to

\footnotetext{
${ }^{1}$ Note that there are different definitions in which the sequence of the rotational elements corresponds to the sequence of the elemental rotations, e.g. $z-y-x$. This, however, contradicts to (1), assuming that $\mathbf{J}$ is computed by (3).
}

the direction of flow. But such setup-dependent approaches go beyond the scope of this paper.

There are approaches as [20] that take into account that the environmental contact might be viscoelastic. This is especially advantageously with soft contact, as in medical applications. In industrial scenarios however, a purely elastic environment is sufficient, even more if other terms of the robot model are neglected, as in section II-A.

\section{Modeling of the Whole Setup}

The total setup is then modeled by

$$
\Delta \mathbf{x}=\Delta \mathbf{x}_{j}+\Delta \mathbf{x}_{s}+\Delta \mathbf{x}_{e}=\mathbf{C f}=\left(\mathbf{C}_{j}+\mathbf{C}_{s}+\mathbf{C}_{e}\right) \mathbf{f} .
$$

Fig. 3 shows the notation with a stiff sensor. ${ }^{2}$ It is quite evident that Cartesian measurements of the tcp pose in place of the pose that is computed from measured joint values are not an option, since then the real and the measured position would coincide so that only $\Delta \mathrm{x}_{e}$ would remain.

\section{E. Identification of the Parameters}

The identification of the parameters depends on the setup. For a setup with a stiff sensor, a concentrated environmental compliance and no additional sources of compliance, only the diagonal elements of the matrices $\mathbf{C}_{q}$ and $\mathbf{C}_{e}$ have to be computed. When pressing vertically against a table (as in Fig. 5), only the deflection of the normal position component is significant, since the other elements are zero, besides friction. This means, even with tilting the end-effector, only the position components of $\mathbf{C}_{e}$ can be identified. So the measurement equation is

$$
z_{e}=z+\mathbf{j}_{z}^{T} \mathbf{C}_{q} \mathbf{J}^{T} \mathbf{f}+\mathbf{c}_{e z}^{T} \mathbf{f}=z+\mathbf{c}_{z}^{T} \mathbf{f},
$$

where $\mathbf{j}_{z}^{T}, \mathbf{c}_{e z}^{T}$, and $\mathbf{c}_{z}^{T}$ are the third rows of $\mathbf{J}, \mathbf{C}_{e}$, and $\mathbf{C}$.

Equation (18) is linear in the parameters and the object pose. Therefore a least squares algorithm can be applied. For a fixed contact point $\left(x_{e}, y_{e}, z_{e}\right)^{T}$, the robot is slightly moved vertically by Cartesian commands, in order to measure the forces at a couple of positions. Because of the static model this can be done step by step. The robot position $\left(x=x_{e}, y=y_{e}, z\right)^{T}$ is computed from the measured encoder values at the joint motors, i.e. before the joint compliance. In this way the local compliance $\mathbf{c}_{z}\left(\mathbf{x}_{e}\right)$ (and the contact point coordinate $z_{e}$ ) can be identified. When moving to further contact points with different Jacobians, the compliance parameters $\mathbf{C}_{q}$ and $\mathbf{c}_{e z}$ can be estimated.

This is easier if the robot postures are chosen to show zero elements in $\mathbf{j}_{z}^{T}$, as in the left hand side of Fig. 5 where only joints 2 and 3 are involved. Furthermore, for numerical reasons, it is advisable to scale the compliance parameters, since they are in different orders of magnitude.

Instead of a single identification of the parameters, they can be adapted during control, as in [21]. In view of the wide stability range, however, this is not needed with the control approach of section III.

\footnotetext{
${ }^{2}$ Note that there are different definitions in which both, $\mathbf{f}$ and $\Delta \mathbf{x}$, have another sign.
} 


\section{F. Implementation}

In the current implementation, three details are different from the so far explained method:

- The forces and torques are represented by sensor values, not by a vector in the world frame

The measured forces and torques ${ }_{s} \mathbf{f}$ have to be transformed in two ways. First, they have to be related to the tcp, instead of the sensor. This is done by (6) and (7). Second, the vector ${ }_{t} \mathbf{f}$ has to be rotated, such that it is expressed with respect to the world frame. This is done by a multiplication with $\mathbf{R}^{\prime}$, compensating the effect of $\mathbf{R}^{\prime T}=\mathbf{R}^{\prime-1}$ in (15).

- The Jacobian is expressed with respect to the tcp frame The Jacobian is computed similar to (3) by

$$
\boldsymbol{\Delta}_{t} \mathbf{x}_{j}={ }_{t} \mathbf{J} \boldsymbol{\Delta} \mathbf{q}
$$

This is advantageous since the orientational elements in ${ }_{t} \mathbf{x}_{j}$ are small. In this way there are no singularities in the use of the Jacobian.

Then, ${ }_{t} \mathbf{f}$ may be used instead of $\mathbf{f}$ in (1) and with

$$
\boldsymbol{\Delta}_{t} \mathbf{x}_{j}={ }_{t} \mathbf{J} \mathbf{C}_{q t} \mathbf{J}^{T}{ }_{t} \mathbf{f}={ }_{t} \mathbf{C}_{j t} \mathbf{f}
$$

the resulting compliance is expressed in the tcp system.

- The environmental compliance is expressed with respect to the tcp frame

In the configuration of Fig. 5 the end-effector is significantly weaker in cross direction. Therefore the environmental compliance is expressed in the end-effector frame. In other configurations it might be advantageous to express it in the world frame or even to distinguish an end-effector-fixed part and a world-fixed part.

Thus all pose increments and vectors at the tcp are expressed with respect to the end-effector system. This makes the modeling independent from the definition of a world system and results in

$$
\boldsymbol{\Delta}_{t} \mathbf{x}=\boldsymbol{\Delta}_{t} \mathbf{x}_{j}+\boldsymbol{\Delta}_{t} \mathbf{x}_{s}+\boldsymbol{\Delta}_{t} \mathbf{x}_{e}=\left({ }_{t} \mathbf{C}_{j}+{ }_{t} \mathbf{C}_{s}+{ }_{t} \mathbf{C}_{e}\right)_{t} \mathbf{f} .
$$

Then the identification is done using

$$
z_{e}=z+\mathbf{r}_{z}{ }_{z}^{T}\left({ }_{t} \mathbf{J} \mathbf{C}_{q t} \mathbf{J}^{T}+{ }_{t} \mathbf{C}_{e}\right)_{t} \mathbf{f},
$$

where $\mathbf{r}_{z}^{\prime T}$ is the third row of $\mathbf{R}^{\prime}$.

\section{FORCE CONTROL}

The model of (17) can be used to design a force control law which is advantageous with position controlled robots.

\section{A. Position-Based Approach}

The fundamental equation is

$$
\mathbf{x}_{d}=\mathbf{x}+\mathbf{C}\left(\mathbf{f}-\mathbf{f}_{d}\right)=\mathbf{x}_{e}-\mathbf{C f}_{d} .
$$

Here, $\mathbf{x}_{d}$ is the desired pose which is computed from the actual pose of the tcp $\mathbf{x}$ and the force control error $\left(\mathbf{f}-\mathbf{f}_{d}\right)$ that is measured simultaneously with the pose (see Fig. 4). $\mathbf{x}_{e}$ is the (a priori unknown) contact pose (see Fig. 3).

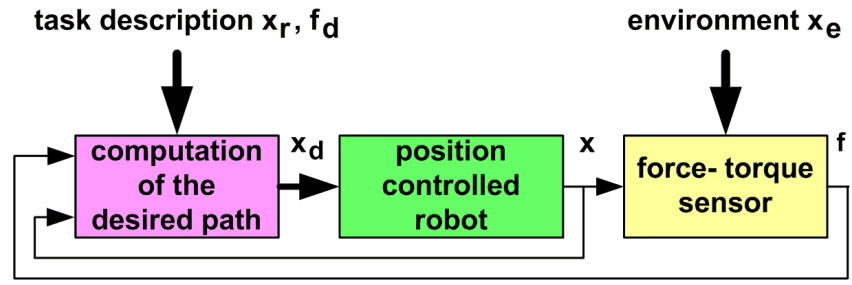

Fig. 4. Control architecture with a position controlled robot and the computation of the desired path from sensed forces

Equation (23) differs from usual approaches ${ }^{3}$ as

$$
\mathbf{x}_{c}(k)=\mathbf{x}_{c}(k-1)+\mathbf{K}\left(\mathbf{f}(k)-\mathbf{f}_{d}\right)
$$

which increment a pose command $\mathbf{x}_{c}$.

The crucial difference is that in (23) no pose is incremented but a geometrical relation is described, which is valid at any time, so that a time index $k$ is not required. This means that a single ${ }^{4}$ measurement in contact is sufficient to compute a desired pose which results in the desired force, assuming a time-invariant environment and perfect knowledge of the compliance.

Another difference is that the identified compliance $\mathbf{C}$ of the total system can be used directly. So there is no control gain $\mathbf{K}$ required since (23), strictly speaking, does not represent a controller. Therefore, (23) is also denoted as the computation of the desired pose.

The resulting control performance is then exclusively given by the position controller. As a precaution, the desired forces and torques can be used for a disturbance feedforward. This allows minimal control errors with a predictable contact, e.g. when following a known contour. In contrast, control errors are inevitable in the case of unexpected forces or torques, e.g. when approaching a contour of unknown location, as in sections IV and VI.

In such cases, mechanical compliance is advantageous in order to reduce the contact forces. The modeling of section II allows the fusion of passive (mechanical) compliance and active compliance (control).

\section{B. Stability Issues}

In order to analyze the stability, using (17), (23) is rewritten as

$$
\begin{aligned}
& \mathbf{x}_{d}=\mathbf{x}+\hat{\mathbf{C}}\left(\mathbf{f}-\mathbf{f}_{d}\right)=\mathbf{x}+\hat{\mathbf{C}} \mathbf{C}^{-1}\left(\mathbf{x}_{e}-\mathbf{x}\right)-\hat{\mathbf{C}} \mathbf{f}_{d} \\
& \mathbf{x}_{d}=\tilde{\mathbf{C}} \mathbf{x}+\overline{\mathbf{x}}_{d},
\end{aligned}
$$

with the assumed compliance matrix $\hat{\mathbf{C}}$, the error of the compliance matrix $\tilde{\mathbf{C}}=\mathbf{I}-\hat{\mathbf{C}} \mathbf{C}^{-1}$ and an expression $\overline{\mathbf{x}}_{d}=\hat{\mathbf{C}} \mathbf{C}^{-1} \mathbf{x}_{e}-\hat{\mathbf{C}} \mathbf{f}_{d}$. The latter is constant as $\mathbf{x}_{e}$ is the true environmental pose, not the one estimated from $\mathbf{x}$ and f, using $\hat{\mathbf{C}}$.

\footnotetext{
${ }^{3}$ Equation (24) corresponds to the integral approach $\dot{\mathbf{x}}_{d}(k)=\mathbf{K}(\mathbf{f}(k)-$ $\left.\mathbf{f}_{d}\right)$ which is recommended in [12], [16], [21], [22]. Extended setups, e.g. with PI controllers, have been proposed in [12], [21]. In contrast, PD has a small stability region [16].

${ }^{4}$ Of coarse, high bandwidth force sensing is advantageous also with (23), since then the robot can react instantaneously on sudden changes. This applies to a moving environmental pose, e.g. during contour following.
} 
Then, the closed loop transfer function $\mathbf{G}(z)$ of the position control is taken, resulting in

$$
\begin{aligned}
& \mathbf{x}(z)=\mathbf{G}(z) \mathbf{x}_{d}(z)=\mathbf{G}(z) \tilde{\mathbf{C}} \mathbf{x}(z)+\mathbf{G}(z) \overline{\mathbf{x}}_{d}(z) \\
& \mathbf{x}(z)=(\mathbf{I}-\mathbf{G}(z) \tilde{\mathbf{C}})^{-1} \mathbf{G}(z) \overline{\mathbf{x}}_{d}(z) .
\end{aligned}
$$

So, if the assumed compliance matrix is correct, i.e. $\tilde{\mathbf{C}}=\mathbf{0}$, the dynamic characteristics of the position control are adopted without change. On the other side, if $\tilde{\mathbf{C}} \neq \mathbf{0}$, the stable region is reduced. With $\tilde{\mathbf{C}}=\mathbf{I}$, which is true for $\hat{\mathbf{C}}=\mathbf{0}$, and with $\mathbf{G}(z)=z^{-1} \mathbf{I}$, the stability limit of $\left|z_{p}\right|=1$ is reached, where $z_{p}$ are the poles of the transfer function in (26). The same applies for $\tilde{\mathbf{C}}=-\mathbf{I}$, which is true for $\hat{\mathbf{C}}=2 \mathbf{C}$. A delaying dynamical system $\mathbf{G}(z)$ increases the stable range of $\tilde{\mathbf{C}}$ as long as there is no resonance close to the sampling frequency. This is valid for industrial position controllers which typically show no overshooting.

The final value ${ }^{5}$ is computed by

$$
\begin{aligned}
\mathbf{x}_{\infty} & =\lim _{z \rightarrow 1}\left\{(\mathbf{I}-\mathbf{G}(z) \tilde{\mathbf{C}})^{-1} \mathbf{G}(z) \overline{\mathbf{x}}_{d}\right\}=(\mathbf{I}-\tilde{\mathbf{C}})^{-1} \overline{\mathbf{x}}_{d} \\
& =\left(\mathbf{I}-\left(\mathbf{I}-\hat{\mathbf{C}} \mathbf{C}^{-1}\right)\right)^{-1}\left(\hat{\mathbf{C}} \mathbf{C}^{-1} \mathbf{x}_{e}-\hat{\mathbf{C}} \mathbf{f}_{d}\right) \\
\mathbf{x}_{\infty} & =\mathbf{x}_{e}-\mathbf{C} \mathbf{f}_{d}
\end{aligned}
$$

which is not disturbed by an unfavorable assumption of the compliance. Equation (27) is valid, as $\lim _{z \rightarrow 1}\{\mathbf{G}(z)\}=\mathbf{I}$, since all industrial position control statically reaches the desired position.

In contrast to usual stability considerations (as in [16]), here we do not assume a particular position controller, as a PID controller. We only presuppose that the position control is stable and sufficiently damped. In addition, unlike [23], we do not consider reactions on position control due to external forces, since such a feedback is not significant.

Summarizing, (23) results in a stable system, if

1) the underlying position control loop is stable and sufficiently damped,

2) pose as well as forces and torques are measured simultaneously,

3) the identified compliance is not more than twice as much as its real value,

4) all assumptions are valid, e.g. that a static model is sufficient and backslash or friction in the gears is not significant.

It should be noted, that condition 3 is only a rule of thumb which however is conservative ${ }^{6}$. Besides, no stability problem is expected if the compliance is identified too small. This, however, can produce very high control errors, which will be discussed in section IV-A. The exact stability limits can be accessed by computing the poles of (26).

Regarding Fig. 4, the feedbacks of $\mathbf{x}$ and $\mathbf{f}$ cancel each other out if the compliance is assumed properly. So the absence of a real feedback is another explanation that the system is inherently stable. On the other side this means that

\footnotetext{
${ }^{5}$ Strictly speaking, the final value of a signal $y(z)=G(z) x(z)$ is $\lim _{z \rightarrow 1}\left\{\frac{z-1}{z} G(z) x(z)\right\}$ with $x(z)=\frac{z}{z-1} x$ if $x$ is constant.

${ }^{6}$ With decoupled first order transfer functions $G(z)=0.2 z^{-1} /(1-$ $0.8 z^{-1}$ ), e.g., the limit is reached with $\tilde{\mathbf{C}}=-9$ I respectively $\hat{\mathbf{C}}=10 \mathbf{C}$.
}

condition 2 has to be fulfilled for stability. Therefore, in order to reach high performance, we recommend to identify the time-shift between the measurements of position and force.

\section{Effect of the Impact Force}

When approaching an unexpected obstacle from free motion, in the time instant of the impact there will be an additional force which is caused by the inertia of the robot, which has been neglected in section II. However, this impact force can be very high, since the robot and its end-effector are decelerated in a very short time.

With a compliant sensor, it has been computed in [14], that the peak forces can be higher than the other force control errors and therefore can damage the contact surface. With a stiff sensor the problem is tightened because of two differences.

First, the impact force does not only decelerate a compliantly suspended end-effector but as well the rigidly connected robot. Strictly speaking, that part of the robot that is between the deflected joints and the tcp. This results in an even higher risk for the surface. This can only be reduced by decelerating before the impact, e.g. using a camera that predicts the collision.

Second, while a compliant force sensor is located between the end-effector and the robot and therefore does not sense the deceleration force between the end-effector and the obstacle, a stiff sensor measures that part of the impact force that decelerates the robot. Since the impact force is very short, in the order of $1 \mathrm{~ms}$, it can not be sensed reliably. ${ }^{7}$ Anyway, it should not cause any control reaction since the deceleration is neglected in (23).

Therefore, the first sensor value after a collision is discarded and not used to compute the desired pose. Instead, since there is information that a contact is present, as preliminary estimation of the desired pose,

$$
\mathbf{x}_{d}=\mathbf{x}
$$

is set in the first time instant after a collision, if $|\mathbf{f}|>\left|\mathbf{f}_{d}\right|$. Otherwise, (23) ist used.

\section{Parallel Force And Trajectory Control}

For complex tasks, usually desired forces and torques as well as a trajectory of position and orientation is given. But only 6 degrees of freedom (dof) can be controlled independently. There are different strategies to cope with this contradiction.

Impedance control [1] defines the desired trajectory (and thus the desired forces and torques) as a compromise between the given reference trajectory and the given reference forces and torques. This compromise might reduce force errors to a large extent, but it can be unsatisfactory if the reference trajectory lies within an object, because of a wrong environmental model. On the other hand, this approach can work without switching between contact and free motion.

\footnotetext{
${ }^{7}$ Measurements deny a huge force that exceeds the measurement range of the sensor since the frequency response of the mechanical system filters short impulses.
} 
The other strategy is to separate between force controlled and position controlled dof, since, besides friction, forces can only be applied in directions in which no free motion is possible. This approach has been proposed first in [24] as hybrid force and position control.

A third method is to allow motion as well in directions in which forces are measured. This is called parallel control [25]. This is a valid option if compliances are considered instead of the hybrid model.

In this paper it is assumed that the tracking of the reference trajectory is desired as far as the reference forces and torques are not violated. Then, the latter have priority. This assumption e.g. allows moving along a contour when friction is present.

Another application of parallel control is approaching a coarsely known object with given speed before the contact, followed by explicit force control with independently given desired force. Separately from this, a lateral motion can be executed. In contrast to hybrid control, the position control part can incorporate a priori knowledge on the shape of the object for a position feedforward with respect to the force controlled component, as in [5]. This results in

$$
\mathbf{x}_{d}(k+1)=\mathbf{x}_{d}(k)+\mathbf{x}_{r}(k+1)-\mathbf{x}_{r}(k)
$$

where $\mathbf{x}_{r}($.$) represents the reference trajectory and \mathbf{x}_{d}(k)$ is given in time step $k$ by (23).

A problem might occur when approaching an object of only coarsely known location. The first idea is to set the reference trajectory in such a way that there is definitely contact. Then early contact situations have to be analyzed. This is done in section IV-A. The other method is more cautious and feels for the object. This is treated in section IVB.

\section{A. Limitation of the Force Error during Early Contact}

Early contact is no problem for force control according to (29) if the compliance is exactly known. Then the error of $\mathbf{x}_{d}(k+1)$ is bounded by $\mathbf{x}_{r}(k+1)-\mathbf{x}_{r}(k)$. In addition, the position feedforward $\mathbf{x}_{r}(k+1)-\mathbf{x}_{r}(k)$ may be suppressed if contact is detected before the expected contact time.

But if there is uncertainty in the compliance, and therefore because of the stability criterion 3 in section III-B the assumed compliance $\hat{\mathbf{C}}$ is low, an initial velocity $(\mathbf{x}(k)-$ $\mathbf{x}(k-1))$ is almost not being stopped when contact is detected, as

$$
\mathbf{x}_{d}(k)=\mathbf{x}(k)+\hat{\mathbf{C}}\left(\mathbf{f}(k)-\mathbf{f}_{d}\right) \approx \mathbf{x}(k)
$$

instead of

$$
\mathbf{x}_{d}(k)=\mathbf{x}_{e}-\mathbf{C f}_{d}=\text { const. }
$$

Therefore, unlike (30), $\mathbf{x}_{d}$ is kept constant after the first validly sensed force (i.e. after the second step with contact, cf. section III-C), until the previously expected time of contact. Then, at the latest, the initial motion will be stopped so that a small but nonzero $\hat{\mathbf{C}}$ reduces the control error. So long a maybe raised force, as in the second sampling step after the impact, has to be tolerated. Alternatively, $\mathbf{x}_{d}$ can be filtered during this phase.

\section{B. Searching for Contact}

The more cautious method is to stop the approaching motion before an expected contact and then to increment the desired pose $\mathbf{x}_{d}$ and the expected location of the environment $\hat{\mathbf{x}}_{e}$ by a predefined searching step, if

$$
\mathbf{x}^{T} \mathbf{C} \mathbf{f}_{d}\left(\mathbf{f}_{d}^{T} \mathbf{C}^{T} \mathbf{C} \mathbf{f}_{d}\right)^{-1 / 2}<\hat{\mathbf{x}}_{e}^{T} \mathbf{C} \mathbf{f}_{d}\left(\mathbf{f}_{d}^{T} \mathbf{C}^{T} \mathbf{C} \mathbf{f}_{d}\right)^{-1 / 2}
$$

This means that searching is done if the projection of the pose of the tcp in the direction of the desired deflection $\mathbf{C f}_{d}$ is smaller than the projection of the expected environmental pose, in other words, if a contact at $\mathbf{x}_{e}$ would be sensed (see Fig. 3).

Unfavorable with this method is that after the execution of a searching step the robot has to wait until the new desired pose is (almost) reached, before the next step is commanded. So this approach is quite slow. Even more since a medium size searching step statically may result in a large force.

In addition, force controlled moving without a sensed force is always dangerous. So a maximum searching length has to be defined, as a precaution.

Therefore the reference trajectory is chosen in such a way that there is definitely contact and searching is only used to find back to the environment if contact is lost due to an excessive reaction on the measured force.

\section{CONSIDERATION OF COUPLINGS AND OF PARTIALly CONSTRAINED CONFIGURATIONS}

The problem of couplings between forces and torques has already been discussed in [14] for compliance in the sensor. It means that in order to exert a force at the tcp, in general, in the sensor both a force and a torque is required. This is solved by the computation of ${ }_{t} \mathbf{f}$ from (6) and (7). It results in the desired final pose (see Fig. 2), if no noise is present.

Another point is that ${ }_{t} \mathbf{C}$ is not diagonal, neither ${ }_{t} \mathbf{C}_{j}$ nor ${ }_{t} \mathbf{C}_{s}$. For compliance in the robot joints the effect is known by the difference between the Jacobian transpose and the inverse Jacobian. While the latter will result in the desired Cartesian motion in the unconstrained case, the transpose, as in (1) and therefore in (4), produces the desired forces and torques, incorporating deflections in the robot joints. This results in a direct motion to the desired pose. So the compensation of couplings by high-bandwidth control as proposed by [26] is not required. Couplings are sufficiently compensated.

But (23) is only valid for a fully constrained contact, in which 6 dof of the transformation from the end-effector system to the environment are represented by forces and torques. In contrast, in partially constrained configurations it might be advantageous to detect which elements of the pose vector are given by the forces and torques. For example in a vertex-face contact, in which a small tip presses against a plain object as in the right hand side of Fig. 2, the orientation of the object is not constrained and thus not sensible. In addition, with a small friction coefficient the lateral position components are uncertain as well, so that only the normal component is informative. This means that, in order to prevent drifting due to noise, only the constrained dof, the normal direction, is controlled by force. The other 

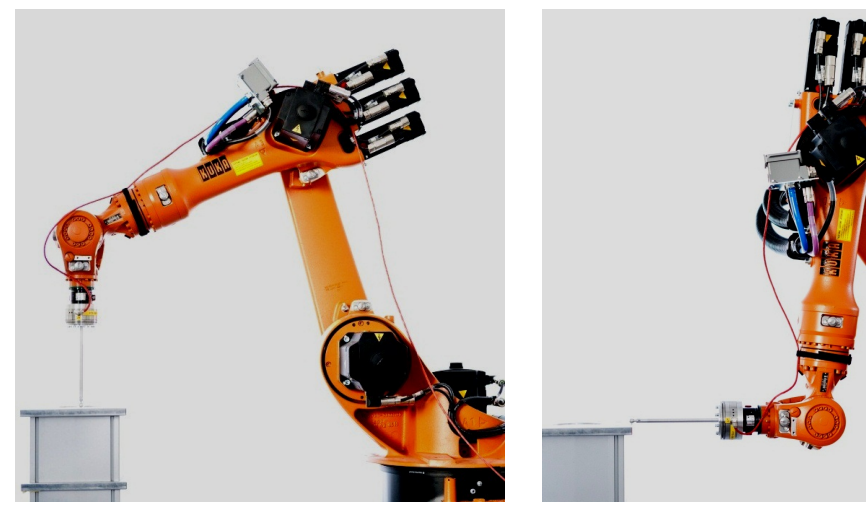

Fig. 5. Force control experiments with a stiff sensor and a fixed environment

components are purely position controlled with a given reference trajectory, as in hybrid force and position control. This can be expressed by

$$
\begin{aligned}
& \mathbf{x}_{d}(k+1)= \\
& \left\{\begin{array}{cl}
\mathbf{x}_{d}(k)+\mathbf{x}_{r}(k+1)-\mathbf{x}_{r}(k) & \text { sensed components } \\
\mathbf{x}_{r}(k+1) & \text { other components }
\end{array}\right.
\end{aligned}
$$

\section{EXPERIMENTS}

The experiments are implemented at a smaller setup (see Fig. 5) since it is hard to interpret experiments with the setup of Fig. 1 because of nonmodeled effects. By the way, the proposed approach is not only intended for moving belt tasks. Instead it may be used in generic force control.

The experimental setup consists of a KUKA KR16 robot with a KR C4 controller [27] (Fig. 5). The sensor is an own construction denoted by FTS-100, which communicates with the robot controller via EtherCAT ${ }^{\circledR}$, the preferred interface of the KR C4. Using strain gauges the system is classified as a stiff 6 dof force- / torque-sensor with a range of $800 \mathrm{~N}$ and $80 \mathrm{Nm}$.

In the experiments the robot presses vertically onto the table since otherwise the table or the tip might slip. With a vertical tip the system is quite stiff, with ${ }_{t} c_{z} \approx 0.003 \mathrm{~mm} / \mathrm{N}$, while a horizontal tip features ${ }_{t} c_{x y} \approx 0.030 \mathrm{~mm} / \mathrm{N}$. For comparison, ${ }_{t} \hat{c}_{z}=0.024 \mathrm{~mm} / \mathrm{N}$ was used in [14] because of the compliant sensor.

Nevertheless, even in the stiff configuration according to the left hand side of Fig. 5, the experiment of approaching the table with $20 \mathrm{~mm} / \mathrm{s}$ and controlling the force when contact is detected is executed with a similar performance as with the compliant sensor (Figs. 6 and 7, note the differing reference forces and scaling). The remaining oscillation, just after the overshooting, is due due to neglecting the dynamical effects stated in condition 4 in section III-B. This is e.g. the mass of the robot links that increases the joint deflection during accelerations. Compared with a standard PI-controller, the proposed setup is superior, which has already been shown in [14]. Fig. 8 shows an experiment with the lower stiffness of a lateral contact, touched with $50 \mathrm{~mm} / \mathrm{s}$. Here, a higher noise is present, which results from the bigger measurement range of the new sensor.

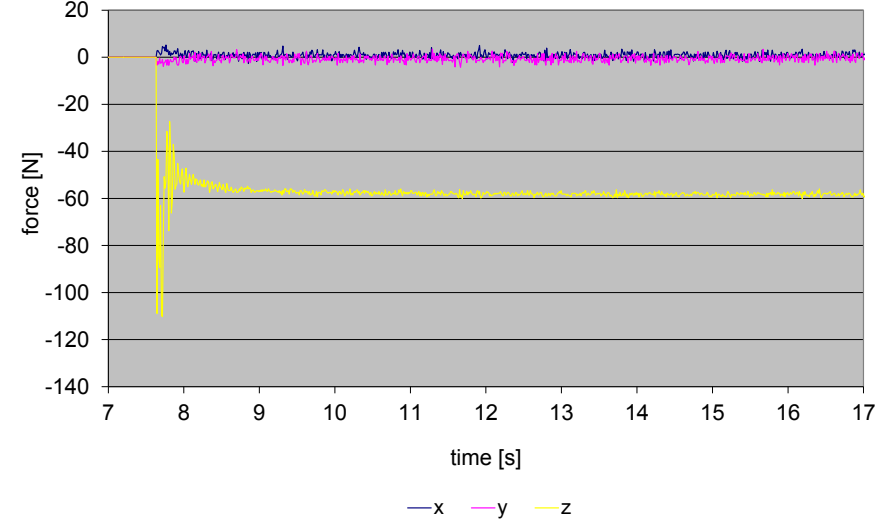

Fig. 6. Measured force during the experiment according to the left hand side of Fig. 5

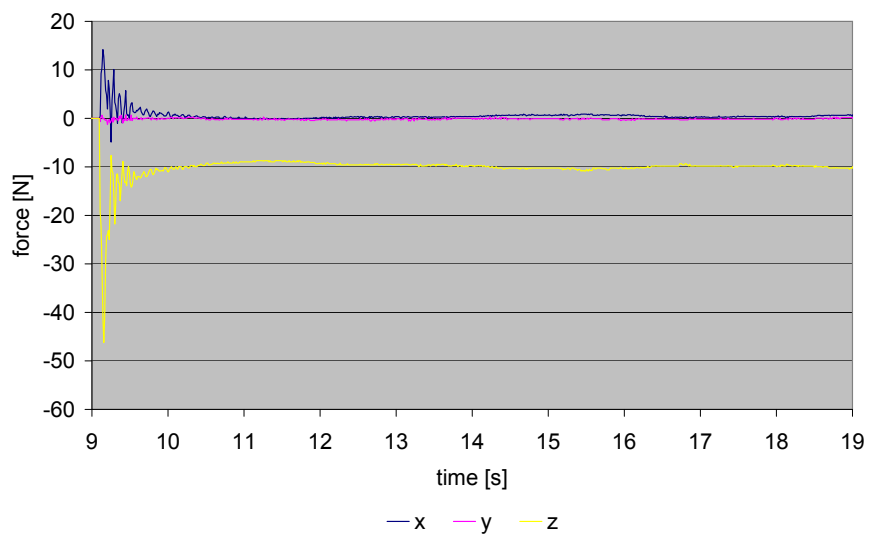

Fig. 7. Measured force during the experiment with a compliant sensor [14]

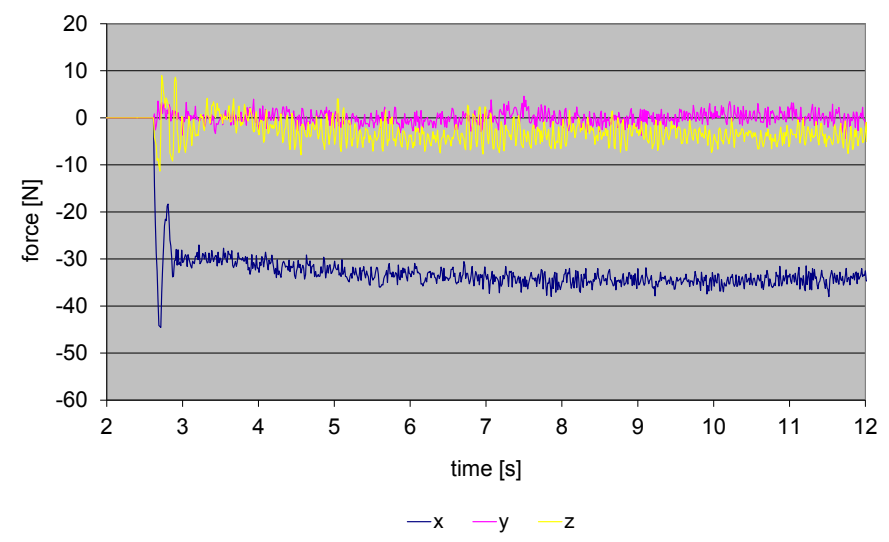

Fig. 8. Measured force during the experiment according to the right hand side of Fig. 5

\section{CONCLUSION}

A method for position-based control of forces and torques has been presented. It is advantageous especially with position controlled robots as typical industrial robots, regardless of the type of sensor.

In contrast to the previous paper [14], there is no more a compliant sensor required. But a compliance somewhere in the system is mandatory, since position-based force control is 
not possible else. And the compliance has to be reproducible, in order to be modeled or identified.

With respect to previous work of other authors, it is crucial that

- we process the measured position instead of the previous commanded values, ${ }^{8}$

- we use a geometric interpretation which is inherently stable and independent of the robot dynamics,

When approaching an object of unknown location, the desired force is reached and maintained very fast. The control performance is not affected by delays or noise in the sensor data.

\section{FUTURE WORK}

So far, the fundamental experiment of controlling a constant desired force at a constant pose is solved. Future experiments will include time variant desired forces $\mathbf{f}_{d}(k)$ or motion $\mathbf{x}_{r}(k)$ tangential to the contact surface (as e.g. in contour following or polishing). These experiments do not require extensions in the force control algorithm. Instead, they will exhibit disturbances which can hardly be suppressed, as the impact in the shown experiments. The resulting performance will depend on position control and on the accuracy of the assumed parameters.

This may be a reason for an adaptation of the compliance as in [21] and for improvements in position control. The latter does so far neither consider the robot joint compliance nor any limitation of the acceleration or the motor torque. Strictly speaking, the used position control is designed only for motion without contact, which is the more critical mode in [17].

But not only position control can be improved. Backslash and friction in the gears are not considered for force control. As well, the mass of the robot links and the damping within the gears or the environment are not included in (23). Their consideration in the force control law (23) seems more important than in position control.

Finally, a new tool for the wheel assembly task of Fig. 1 can be designed, using a stiff sensor and thus less deflections. Experiments will probably demonstrate that the presented control approach is valid as well for moving belt production without a designed significant mechanical compliance.

\section{REFERENCES}

[1] N. Hogan. Stable execution of contact tasks using impedance control. In Proc. 1987 IEEE Int. Conf. on Robotics and Automation (ICRA), pages 1047-1054, Raleigh, NC, USA, March 1987.

[2] A. Albu-Schäffer, C. Ott, and G. Hirzinger. A unified passivitybased control framework for position, torque and impedance control of flexible joint robots. The Int. Journal of Robotic Research, 26(1):2339, 2007.

[3] G. Ganesh, N. Jarrassé, S. Haddadin, A. Albu-Schäffer, and E. Burdet. A versatile biomimetic controller for contact tooling and haptic exploration. In Proc. 2012 IEEE Int. Conf. on Robotics and Automation (ICRA), pages 3329-3334, St. Paul, MN, USA, May 2012.

[4] K. Lee and M. Buss. Force tracking impedance control with variable target stiffness. In Proc. of the 17th IFAC World Congress, pages 6751-6756, Seoul, Korea, July 2008.

\footnotetext{
${ }^{8}$ In the early 1990 s, when most of the methods were designed, common interfaces of industrial robots did not provide the measured position.
}

[5] A. Spiller and A. Verl. Superimposed force/torque-control of cooperating robots. In Proc. Joint 41th Int. Symp. on Robotics and 6th German Conf. on Robotics ISR/ROBOTIK 2010, Munich, Germany, June 2010.

[6] D. E. Whitney and J. L. Nevins. What is a remote center compliance (RCC) and what can it do? In 9th Int. Symposium on Industrial Robots, Washington D. C., USA, 1979.

[7] G. Reinhart and J. Werner. Flexible automation for the assembly in motion. Annals of the CIRP, 56(1):25-28, 2007.

[8] J. Grewe, G. Hirzinger, R. Koeppe, C. Strobl, and B. Willberg. Compliant-force-torque-sensor. In Proc. 10th Int. Workshop on Robotics in Alpe-Adria-Danube Region, Vienna, Austria, May 2001.

[9] SCHUNK GmbH \& Co. KG Spann- und Greiftechnik. Measuring. http://www.schunk. com/schunk/schunk_websites / products/products_level_3/product_level3. html ?product_level_3=296\&product_level_2= $252 \&$ product_level_1 $=244 \&$ country $=\bar{I}$ NT $\& 1$ ng Code $=$ $\mathrm{EN} \& \mathrm{lngCode} 2=\mathrm{EN}$, last visited 2012.

[10] ATI Industrial Automation. Multi-axis force / torque sensors. http: //www.ati-ia.com/products/ft/sensors.aspx, last visited 2012

[11] L. Villani and J. de Schutter. Force control. In Handbook of Robotics, pages 161-185. Springer, 2008.

[12] J. De Schutter and H. Van Brussel. Compliant robot motion II. A control approach based on external control loops. The Int. Journal on Robotics Research, 7(4):18-33, 1988.

[13] F. Caccavale, C. Natale, B. Siciliano, and L. Villani. Integration for the next generation: Embedding force control into industrial robots. IEEE Robotics and Automation Magazine, 12(3):53-64, 2005.

[14] F. Lange, C. Jehle, M. Suppa, and G. Hirzinger. Revised force control using a compliant sensor with a position controlled robot. In Proc. 2012 IEEE Int. Conf. on Robotics and Automation (ICRA), pages 1532-1537, St. Paul, MN, USA, May 2012.

[15] D. Navarro-Alarcon, P. Li, and H. M. Yip. Energy shaping control for robot manipulators in explicit force regulation tasks with elastic environments. In Proc. 2011 IEEE/RSJ Int. Conf. on Intelligent Robots and Systems (IROS), pages 4222-4228, San Francisco, CA, USA, Sept. 2011

[16] P. Rocco, G. Ferretti, and G. Magnani. Implicit force control for industrial robots in contact with stiff surfaces. Automatica, 33(11):20412047, 1997.

[17] F. Lange, J. Werner, J. Scharrer, and G. Hirzinger. Assembling wheels to continuously conveyed car bodies using a standard industrial robot. In Proc. 2010 IEEE Int. Conf. on Robotics and Automation (ICRA), pages 3863-3869, Anchorage, AK, USA, May 2010.

[18] H. Chen, W. Eakins, J. Wang, G. Zhang, and T. Fuhlbrigge. Robotic wheel loading process in automotive manufacturing automation. In Proc. 2009 IEEE/RSJ Int. Conf. on Intelligent Robots and Systems (IROS), pages 3814-3819, St. Louis, USA, Oct. 2009.

[19] IBG - Goeke Technology Group. Wheel assembly. http: //www goeke-group. com/index.php? id=56\&L=4, last visited 2012.

[20] P. Moreira, C. Liu, N. Zemiti, and P. Poignet. Soft tissue force control using active observers and viscoelastic interaction model. In Proc. 2012 IEEE Int. Conf. on Robotics and Automation (ICRA), pages 4660-4666, St. Paul, MN, USA, May 2012.

[21] J. Roy and L. L. Whitcomb. Adaptive force control of position/velocity controlled robots: theory and experiment. IEEE Trans. on Robotics and Automation, 18(2):121-137, 2002.

[22] B. R. Shetty and M. H. Ang Jr. Active compliance control of a PUMA 560 robot. In Proc. 1996 IEEE Int. Conf. on Robotics and Automation (ICRA), pages 3720-3725, Minneapolis, MN, USA, April 1996.

[23] G. Ferretti, G. Magnani, and P. Rocco. Toward the implementation of hybrid position/force control in industrial robots. IEEE Trans. on Robotics and Automation, 13(6):838-845, 1997.

[24] M. H. Raibert and J. J. Craig. Hybrid position / force control of manipulators. Trans. of the ASME, 102, 1981.

[25] S. Chiaverini and L. Sciavicco. The parallel approach to force/position control of robotic manipulators. IEEE Trans. on Robotics and Automation, 9(4):361-373, 1993

[26] T. Olsson et al. Cost efficient drilling using industrial robots with high-bandwidth force feedback. Robotics and Computer-Integrated Manufacturing, 26:24-38, 2010.

[27] KUKA Roboter GmbH. KUKA industrial robots - KR C4. http://www.kuka-robotics.com/en/products / controllers/kr_c4/, last visited 2012. 